\title{
The New Molecular Entity Evolocumab, One Kind of PCSK9 Inhibitor, Reduce Plasma Small Size LDL-Cholesterol Levels by Using a New Standardized Method of Measuring LDL Size
}

\author{
Ikuo Inoue ${ }^{1 *}$, Ryo Kubota ${ }^{2}$, Shohan Yanagi ${ }^{3}$, Masumi Akita4, Takanari Nakano5, \\ Shigehiro Katayama1, Akira Shimada', Mistuhiko Noda1 \\ ${ }^{1}$ Department of Endocrinology and Diabetology, Saitama Medical University, Saitama, Japan \\ ${ }^{2}$ Department of Health Sciences, Saitama Prefectural University, Saitama, Japan \\ ${ }^{3}$ Research and Development Department, Advanced Materials Development Sector, Tokyo Metropolitan Industrial Technology \\ Research Institute, Tokyo, Japan \\ ${ }^{4}$ Division of Morphological Science, Biomedical Research Center, Saitama Medical University, Saitama, Japan \\ ${ }^{5}$ Department of Biochemistry, Saitama Medical University, Saitama, Japan \\ Email: ^i1901018@saitama-med.ac.jp, kubota-ryo@spu.ac.jp, yanagi.syouhan@iri-tokyo.jp, makita@saitama-med.ac.jp, \\ nk.takanari@gmail.com, skataya@saitama-med.ac.jp, asmd@saitama-med.ac.jp,noda-3im@io.ocn.ne.jp
}

How to cite this paper: Inoue, I., Kubota, R., Yanagi, S., Akita, M., Nakano, T., Katayama, S., Shimada, A. and Noda, M. (2017) The New Molecular Entity Evolocumab, One Kind of PCSK9 Inhibitor, Reduce Plasma Small Size LDL-Cholesterol Levels by Using a New Standardized Method of Measuring LDL Size. Open Journal of Molecular and Integrative Physiology, 7, 1-23. https://doi.org/10.4236/ojmip.2017.71001

Received: January 25, 2017

Accepted: February 25, 2017

Published: February 28, 2017

Copyright ( 92017 by authors and Scientific Research Publishing Inc. This work is licensed under the Creative Commons Attribution International License (CC BY 4.0).

http://creativecommons.org/licenses/by/4.0/

\section{c) (i) Open Access}

\begin{abstract}
Aims: There has been no evidence on the effects of evolocumab, protein convertase subtilisin/kexin type 9 (PCSK9) inhibitor, on small size LDL. We observationally investigated the efficacy and side effects of evolocumab on the LDL subfraction particle diameter using PAGE system for lipoprotein analysis. Methods: We defined 30 patients with high-risk hyperlipidemia. As for analysis of LDL subfraction profile, we used polyacrylamide gel electrophoresis three methods: 1) 3\% nondenatured poly-acrylamide gel electrophoresis method (3\%PAGE), 2) $2 \%$ - 16\% nondenatured poly-acrylamide gradient gel electrophoresis method (2\% - 16\% GGE) and 3) 2.7\% - 5\% GGE. Evolocumab $140 \mathrm{mg} /$ day administered together with statin significantly improved serum total cholesterol (TC), triglyceride (TG), high-dense lipoprotein-cholesterol (HDL-C), and LDL-C after four-week treatment. Results: TC, TG, HDL-C and LDL-C levels were improved by, respectively, $33 \%, 20 \%, 10 \%$, and $54 \%$. The mean LDL size significantly increased from $25.6 \pm 0.4 \mathrm{~nm}$ to $26.4 \pm 0.8 \mathrm{~nm}$. The small dense LDL-cholesterol (sdLDL-C), large buoyant LDL-cholesterol (lbLDL-C), and mid-band lipoprotein-cholesterol were reduced, respectively. Therefore, the preliminary study on this paper can be the first step into a new insight on the world of lipid metabolism. Conclusion: Short-term adminis-
\end{abstract}


tration of evolocumab added-ons to statin therapy, significantly reduced small size LDL levels.

\section{Keywords}

PCSK9 Inhibitor, Evolocumab, LDL Heterogeneicity, Small Size LDL, $3 \%$ PAGE, $2 \%$ - 16\% GGE

\section{Introduction}

Elevated low-density lipoprotein cholesterol (LDL-C) level is an independent risk factor for coronary artery disease (CAD); however, large CAD prevention trials have demonstrated that reducing LDL-C level by intensive 3-hydroxymethylglutaryl CoA (HMG-CoA) reductase inhibitors (statins) treatment regime does not prevent the majority of CAD events [1]. Large-scale intervention studies on the main risk factors of atherosclerosis indicated that lowering the serum total cholesterol (TC) levels by $24 \%-25 \%$ contributes to prevention of only $36 \%$ of total cardiovascular events [2]. In other words, we need to find out how to treat those patients with no improvement from statins. In some cases, we cannot use enough doses of statins due to adverse effects (unmet needs) [3], resulting in its reduced efficiency. In addition to that dilemma, there are still other critical risk factors including hypertension, diabetes, obesity, smoking, high triglyceridemia and hypo high-density lipoprotein-cholesterolemia (hypoHDL-emia).

It is also believed that LDL subpopulations, classified according to size, buoyant density, and chemical composition, have proved essential rather than total LDL-C itself [4] [5]. Many investigators have studied the relationship between the potent pro-atherogenic lipoprotein particles and cardiovascular risks with the aim of improving the prediction of CAD events and identifying patient groups that should be treated [1] [6] [7]. We have paid the most attention to small size, dense high LDL (small, dense LDL (sdLDL)). For example, patients with elevated sdLDL levels have a 2- to 3 -fold increased risk of CAD. Some studies have demonstrated that several statins reduce not only LDL but also sdLDL [8]. However, we have believed that we need to lower sdLDL further [9].

With those backgrounds, we have been long waited for more powerful, new medications to be developed. Evolocumab (AMG 145; Repatha ${ }^{\circledR}$; Amgen Inc., Thousand Oaks, CA), inhibits protein convertase subtilisin/kexin type 9 (PCSK9) recently became available first in the Europe and United States (US) [10], and finally in Japan last year. Evolocumab, a fully human immunoglobulin G2 monoclonal antibody $(\mathrm{mAb})$, mediates proteolytic degradation of hepatic LDL receptors (LDLR) resulting in more efficient clearance of apolipoprotein B (ApoB)containing lipoproteins [10]. However, it has been not reported that the status of the LDL subpopulations is improved by evolocumab treatment. In this study, we investigated that the relationship.

Both "quantity" and "quality" of LDL-C must be clinically valuable; however, 
clinicians usually receive unsatisfactory test results for lipoprotein profiling because of the limited number of available assays for lipids and lipoproteins in clinical laboratories (usually only 3 or 4 items) [11]. Analytical ultracentrifugation, poly-acrylamide gradient gel electrophoresis method [6], nuclear magnetic resonance (NMR), high-performance liquid chromatography (HPLC) with gel filtration columns, ion mobility analysis (IM), and dynamic light scattering (DLS) [12], and homogenous assays, have been used to assess LDL particle sizes [13] [14]. Recently, we developed a novel fluorescence detection method [15] but the methods mentioned above are technically-demanding, expensive, or both. Moreover, there has been no standardization of these LDL-size assessments [16].

In this study, we evaluated the change of the heterogeneity of LDL particles by treatment of evolocumab in 30 patients with high-risk hyperlipidemia, using PAGE system for lipoprotein analysis.

\section{Materials and Methods}

\subsection{Subjects}

The design of this study was prospective, open-labelled, blinded study, and subjects were investigated in October 2015 and evolocumab were treated from $\mathrm{Au}$ gust 2016. Eligible patients were assigned either statin or statin plus evolocumab. We produced the sets of random allocations, by a research support person (who will not be performing data collection) in advance of the start of the study, which were then sealed in consecutively numbered opaque papers. Once the patient has given consent to be included in the trial, the patient was then irreversibly randomised by looking the next sealed papers containing the assignment. Patients in both groups were counselled to follow the National Cholesterol Education Program step I diet. And the patients visited the outpatient clinic of Saitama Medical University Hospital Endocrinology/Diabetes Department.

25 patients were available for statin group. Treatment in the statin group was started at $5 \mathrm{mg}$ per day atorvastatin, or at $2.5 \mathrm{mg}$ per day rosuvastatin. During follow-up, the dose of atorvastatin, or rosuvastatin could be adjusted by the treating physician, with uptitration to $40 \mathrm{mg}$ per day, or $20 \mathrm{mg}$ per day if the LDL-C level did not decrease to the target level or less. Maximally tolerated statin dose is the highest tolerable registered dose of daily statin currently administered to the patient, i.e. rosuvastatin $20 \mathrm{mg}$, atorvastatin $40 \mathrm{mg}$. It was planned to be administered lower doses or the other statins in the case of intolerance according to the investigator's judgment. The case of intolerance have the inability to highest tolerable registered dose of statins because of muscle-related symptoms, interstitial lung disease, thrombotic thrombocytopenic purpura, tendonitis, arthralgia, arthritis, headache, asthenia, dizzy, fatigue, elevation of liver aminotransferases, serum creatinine ( $\mathrm{Cr}$ ) level.

30 patients were available for statin plus evolocumab group. They were treated with atorvastatin (12 patients), rosuvastatin (14 patients), fluvastatin (3 patients), or pravastatin (1 patient). Evolocumab was additionally administered to the patients 1) with $\mathrm{CAD}, 2)$ with heterozygous familial hypercholesterolemia $(\mathrm{HeFH})$, or 3) who were refractory to statin treatment, according to the "2012 
Guidelines for Treatment of Dyslipidemia" by Japan Atherosclerosis Society (JAS). Evolocumab was administered at the dose of $140 \mathrm{mg}$ once every two weeks.

The inclusion and exclusion criteria were described in detail below. Major inclusion criteria were as follows: 1) HeFH, 2) type 2 diabetes mellitus with 120 $\mathrm{mg} / \mathrm{dL}$ or more than $120 \mathrm{mg} / \mathrm{dL}$ LDL-C, 3) patients of "2012 Guidelines for Treatment of Dyslipidemia" by the JAS Category III (Diabetes patients are excepted) with $120 \mathrm{mg} / \mathrm{dL}$ or more than $120 \mathrm{mg} / \mathrm{dL}$ LDL-C, 4) patients of " 2012 Guidelines for Treatment of Dyslipidemia" for secondary prevention with 100 $\mathrm{mg} / \mathrm{dL}$ or more than $100 \mathrm{mg} / \mathrm{dL}$ LDL-C, 5) patients who were refractory to statin treatment, or 6) patients with hypercholesterolemia receiving a statin treatment for at least 6 weeks prior to screening period.

Major exclusion criteria were as follows: patients 1) with receiving insulin, 2) with Glucagon-like peptide 1 (GLP-1) receptor agonists, 3) male, serum $\mathrm{Cr} \geq 3.0$ $\mathrm{mg} / \mathrm{dL}$, woman, serum $\mathrm{Cr} \geq 2.5 \mathrm{mg} / \mathrm{dL}$, 4) fasting triglyceride (TG) is greater than or equal to $400 \mathrm{mg} / \mathrm{dL}$, 5) HbAlc was changed more than $2 \%$ within 3 months, 6) with severe ketosis, diabetic coma or precoma, peripheral artery disease, abdominal aortic aneurysm, carotid artery occlusion $>50 \%$ without symptoms, carotid endarterectomy, carotid artery stent procedure, renal artery stenosis, or renal artery stent procedure, 7) with severe infection, before and after surgery, patients with severe trauma, considered as inadequated by the investigator and doctor, 8) pregnant or breast-feeding women, 9) women of childbearing potential with no effective contraceptive method, 10) participants not previously instructed on a cholesterol lowering diet at the first visit for participants who were being treated with stable dose of statin for at least 6 weeks prior to screening.

For laboratory tests concerning serum lipids, fasting blood samples were collected once every two weeks. TC levels and TG levels were measured by the enzyme method and HDL-C by the selective solubilization method at the facilities at Saitama Medical University. Measurement using 3\% poly-acrylamide gel disc electrophoresis (PAGE) was performed later by MCL (Ltd. Musashi Clinical Laboratories, 309-8 Ooazakamifuzisawa, Iruma-shi, Saitama, Japan). The level of LDL-C was calculated by the method of Friedewald (F method), but patients with TG of $4.5 \mathrm{mmol} / \mathrm{L}(400 \mathrm{mg} / \mathrm{dL})$ or over were excluded.

\subsection{Ethics Statement}

This study was conducted in accordance with the Good Clinical Practice (GCP), International Conference on Harmonization Guidelines (ICH), and applicable laws and regulations. The study protocol was approved by the Ethics Committee of Saitama Medical Hospital. After receiving a full explanation of the study, all patients provided written, informed consent before enrollment. This study is registered with the University Hospital Medical Information Network Clinical Trials Registry (UMIN-CTR; Japan), number UMIN000018818.

\subsection{Nondenaturing Poly-Acrylamide Gradient Gel Electrophoresis (GGE)}

Mean LDL particle diameter was determined by 2\% - 16\% GGE (Gellex Interna- 
tional Co. Ltd. Tokyo, Japan) according to the golden standard method of Nichols, Krauss, and Musliner [6]. Briefly, $10 \mu \mathrm{L}$ of plasma from each subject was diluted 4:1 with a $20 \%$ sucrose/tracking solution and electrophoresed in GGE at $125 \mathrm{~V}$ for 1.5 hours, using a tris(hydroxymethyl)aminomethane $(0.09 \mathrm{~mol} / \mathrm{L}) /$ boric acid $(0.08 \mathrm{~mol} / \mathrm{L}) / \mathrm{Na} 2 \mathrm{EDTA}(0.003 \mathrm{~mol} / \mathrm{L})$ buffer at $\mathrm{pH}$ 8.3. In the present study, we also used the other, narrow-range $2.7 \%$ - 5\% GGE (Gellex International Co. Ltd. Tokyo, Japan) and 3\% PAGE (LipoPhor AS ${ }^{\circledR}$ ) in order to accurately determine the LDL particle diameters, in addition to the broad-range $2 \%-16 \%$ GGE (golden standard method). $2 \%-16 \%$ PAGE is very soft and fragile as compared to $2.7 \%$ - 5\%PAGE. The GGE gels were stained with Oil Red O and scanned using an image scanner, producing a graphical representation of the size distribution of lipoprotein particles.

\subsection{3\% Poly-Acrylamide Gel Disc Electrophoresis (PAGE) and Densitometric Analysis}

3\% PAGE and densitometric analysis were determined by the standard method [17] [18] [19]. Briefly, serum samples (25 $\mu$ l) were added to $200 \mu \mathrm{l}$ of a loading gel solution containing Sudan Black B and injected into a 3\% PAGE gel. The gel was photopolymerized for $30 \mathrm{~min}$, and the loaded samples were electrophoresed for $25 \mathrm{~min}$. The resulting electrophoresed patterns were scanned with a densitometer (Densitron Finger Printer, Jokoh, Japan), and the Area Under the Curve (AUC)\% for the very low-density lipoprotein (VLDL), LDL, and HDL peaks were calculated. The AUC\% values of mid-band lipoprotein and small LDL were identified according to a report by Mishima and colleagues [20] [21] with slight modifications. First, the peak positions of VLDL and HDL were set as 0 and 1 relative migration $(\mathrm{Rm})$. Second, the scanned spectrum was overlaid with a control spectrum representing normal lipoproteins. Finally, the presence or absence of small LDL and mid-band lipoproteins were determined by identifying the excess area in the spectrum of samples on either or both sides of the control LDL peak. Although there were marked changes in the lipid profiles, there are cases in which the changes of the mean LDL sizes were not big. "Quantity" and "quality" of lipoproteins are clinically valuable. According to our previous method [9], we detected eight fractions by this Gaussian fitting method: VLDL1, VLDL2, IDL, L-LDL, M-LDL, s-LDL, vs-LDL, and HDL fractions in order to determine the "quantity" and "quality" of lipoproteins. The intermediate-density lipoprotein (IDL) fraction consistent with mid-band lipoprotein particle. The L-LDL (large size LDL) and M-LDL (medium size LDL) fractions correspond to large buoyant LDL (lbLDL) particles, whereas the s-LDL (small size LDL) and vs-LDL (very small size LDL) fractions correspond to sdLDL particles. Properties of lbLDL particles and sdLDL particles are described below.

\subsection{Determination of sdLDL-C, IbLDL-C, and Mid-Band Lipoprotein-C by 3\% PAGE}

If there were substantial areas for mid-band lipoprotein and/or small LDL, we 
used the AUC\% of Rm > 0.40 and $\mathrm{Rm} 0.10$ to 0.18 in the LDL peak as small LDL and mid-band lipoproteins, respectively [9] [11] [20] [21]. The LDL-C value (total LDL-C) was calculated from the Friedewald equation. The value multiplied by the respective AUC\% of the LDL-C value (total LDL-C) was used as the calculated value of each LDL-C subfraction. The ratios (\%) of sdLDL-C and mid-band lipoprotein-C to total LDL-C were determined. The levels of lbLDLcholesterol (lbLDL-C) were calculated by subtracting sdLDL-C and mid-band lipoprotein-C levels from total LDL-C [9] [11] [22] [23].

\subsection{ApoE Phenotyping}

ApoE phenotyping was performed using a commercial kit (Phenotyping Apo E IFE System, Jokoh Co. LTD., Tokyo, Japan). The APOE gene has 3 different alleles, $\varepsilon 2, \varepsilon 3$, and $\varepsilon 4$, which encode 3 common isoforms (E2, E3, and E4), so there are 6 different ApoE phenotypes: E2/2, E2/3, E2/4, E3/3, E3/4, and $\mathrm{E} 4 / 4$.

\subsection{New Method of Constructing Artificial Lipoprotein Markers (Standard Size Markers)}

No standardization of these size assessments has been performed [16]. We invented a novel molecular ruler, making possible the standardized measurement of the size of LDL particles. The standard lane was scanned and a quadratic curve constructed based on the migration distances of four standards of known diameter: a VLDL size marker, an IDL size marker, an LDL size marker, and a small LDL size marker. Briefly, purified gold colloid (Tanaka Kikinzoku Kogyo K.K., Tokyo, Japan) was bound to albumin (Patent Publication No: JP20165953897) to prepare the standard size markers. For the VLDL size marker, we used the $30 \mathrm{~nm}$ gold colloid standard sample (Gold Nanoparticles, Nominal 30 nm Diameter, Reference Material ${ }^{\circledR}$ 8012, National Institute of Standards \& Technology (NIST)). Because it was pure gold colloid, it had no charge. Thus, it did not migrate during PAGE. The diameters of the four standard size markers were confirmed by two methods: 1) transmission electron microscope (JEOL JEM1400, Tokyo, Japan) and 2) DLS (Nano Particle Analyzer SZ-100, HORIBA Co. Ltd. Kyoto, Japan). DLS measurements were performed at room temperature $\left(25.0^{\circ} \mathrm{C}\right)$ with the green laser output at $532 \mathrm{~nm}$ and the measurement scattering angle set to $90.0^{\circ} \mathrm{C}$. Mean LDL particle diameters were estimated from the standard curve obtained from the relationship between the diameters of the size markers and the $\mathrm{Rm}$ values in PAGE.

\subsection{Statistical Analyses}

Statistical analyses were performed using SPSS 15.0 software (SPSS Statistics 21, IBM). All serum lipids were compared by paired $t$-test with significance levels of $5 \%$ on two sides. The measurement values were expressed in mean \pm SD. Correlation between variables was determined using Spearman's Correlation coefficients. 


\section{Results}

\subsection{Patients' Backgrounds}

Table 1 shows the backgrounds of the patients who were investigated in the study. They were aged 35 - 75 years (mean 60.5 years), 13 men and 17 women, 30 in total, and their mean body mass index was $24.8 \mathrm{~kg} / \mathrm{m}^{2}$. Diseases concurrently present were diabetes mellitus in $16.6 \%$ and hypertension as the primary disease in $10 \%$. Before the initiation of the evolocumab treatment, the mean TC was $5.772 \mathrm{mmol} / \mathrm{L}(223.2 \mathrm{mg} / \mathrm{dL})$, the mean LDL-C was $3.543 \mathrm{mmol} / \mathrm{L}(137.0$ $\mathrm{mg} / \mathrm{dL})$, the mean TG was $1.877 \mathrm{mmol} / \mathrm{L}(167.6 \mathrm{mg} / \mathrm{mL})$, and the mean HDL-C was $1.461 \mathrm{mmol} / \mathrm{L}(56.5 \mathrm{mg} / \mathrm{dL})$. In laboratory data, clinically significant changes in liver and kidney function tests were not detected during the evolocumab treatment. In statin plus evolocumab group, we enrolled 30 patients who had E3/3, E2/3, APOE phenotype. The three bands of apoE3/3 homozygotes are obvious. If there were $\mathrm{E} 4 / 3, \mathrm{E} 2 / 4$, APOE phenotype, we repeated the experiment several times and made sure the E4/3, E2/4, APOE phenotype.

As data not shown, there was not a significant difference between statin group and statin plus evolocumab group in the backgrounds of the patients. There were many cases of inability to highest tolerable registered dose of statins be-

Table 1. Baseline clinical characteristics before the initiation of evolocuma of patients who investigated in the study.

\begin{tabular}{|c|c|c|}
\hline $\mathrm{n}$ & 30 & \\
\hline gender (male/female) & $(13 / 17)$ & \\
\hline age (years) & $60.5 \pm 9.5$ & \\
\hline body mass index & 24.8 & \\
\hline $\mathrm{eGFR}\left(\mathrm{mL} / \mathrm{min} / 1.73 \mathrm{~m}^{2}\right)$ & $77.6 \pm 18.6$ & \\
\hline systolic pressure (mm Hg) & $143.6 \pm 13.4$ & \\
\hline diastolic pressure (mm Hg) & $77.9 \pm 8.9$ & \\
\hline diabetes mellitus (\%) & 16.6 & \\
\hline hypertension (\%) & 10 & \\
\hline ischemic heart disease (\%) & 10 & \\
\hline ischemic stroke (\%) & 0 & \\
\hline \multirow[t]{2}{*}{ smokers $(\%)$} & 0 & \\
\hline & $\mathrm{mmol} / \mathrm{L}$ & $\mathrm{mg} / \mathrm{dL}$ \\
\hline $\mathrm{TC}$ & $5.772 \pm 1.492$ & $223.2 \pm 57.7$ \\
\hline LDL-C & $3.543 \pm 0.398$ & $137.0 \pm 15.4$ \\
\hline TG & $1.877 \pm 0.804$ & $167.6 \pm 71.8$ \\
\hline HDL-C & $1.461 \pm 0.398$ & $56.5 \pm 15.4$ \\
\hline $\mathrm{Lp}(\mathrm{a})$ & $197 \pm 151(\mathrm{mg} / \mathrm{L})$ & $19.7 \pm 15.1$ \\
\hline small-dense LDL-cholesterol & $0.303 \pm 0.341$ & $11.7 \pm 13.2$ \\
\hline large-buoyant LDL-cholesterol & $2.927 \pm 1.252$ & $113.2 \pm 48.4$ \\
\hline mid-band LDL-cholesterol & $0.378 \pm 0.150$ & $14.6 \pm 5.8$ \\
\hline
\end{tabular}


cause of side effect such as muscle-related symptoms. Although TC, TG, HDL-C, and LDL-C levels were improved in statin group, there were not reach to significant difference. Moreover, the sdLDL-C, lbLDL-C, and mid-band lipoprotein-C were not significantly changed (data not shown) in statin group.

\subsection{Changes in Serum Lipids by the Add-On Evolocumab Therapy}

Figure 1 shows the notable changes in TC, TG, HDL-C, and LDL-C levels produced by administration of $140 \mathrm{mg}$ evolocumab every two weeks. With the administration of $140 \mathrm{mg}$ evolocumab, serum TC, TG, HDL-C, and LDL-C levels were improved after the start of evolocumab treatment (respectively, $\mathrm{p}=1.0 \times$ $\left.10^{-7}, p=0.073, p=0.104, p=1.0 \times 10^{-6}\right)$. Percent changes of serum TC, TG, HDL-C, and LDL-C levels were respectively reduced by $33 \%, 20 \%, 10 \%$, and $54 \%$. We indicated an example of the lipoprotein densitometric patterns by $3 \%$ PAGE before (Figure 2(a)) and after (Figure 2(b)) the add-on therapy of 140 mg evolocumab. Although there were marked changes in the lipid profiles, the changes of the mean LDL sizes were not big. Thus, we detected eight fractions by this Gaussian fitting method: VLDL1, VLDL2, IDL, L-LDL, M-LDL, s-LDL, vs-LDL, and HDL fractions in order to determine the "quantity" and "quality" of lipoproteins. Figure 3 indicated the evaluation of changes of Figure 2 in the eight fractions before (A) and after (B) the add-on therapy of $140 \mathrm{mg}$ evolocumab by the Gaussian fitting method.

\subsection{Determination of the Diameters of the Size Markers of Purified Gold Colloid}

The diameters of the four standard size markers were determined by transmission electron microscope. The diameters of the purified gold colloid size markers (VLDL size marker, IDL size marker, LDL size marker, and small LDL size marker)

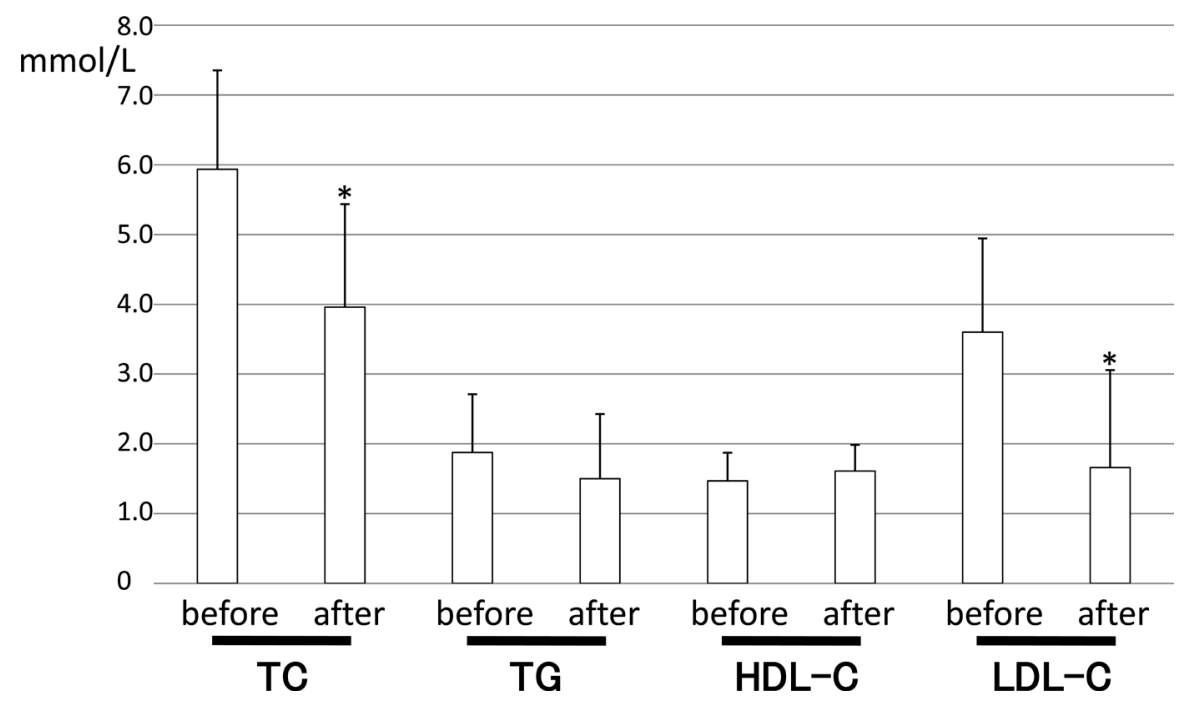

Figure 1. Change of TC, TG, HDL-C, and LDL-C levels before and after the $140 \mathrm{mg}$ evolocumab add-on therapy $(\mathrm{n}=30)$. All values are mean \pm standard deviations. ${ }^{*}$ significantly different from value obtained before evolocumab therapy $(p<0.001$, by paired- $t$ test). 


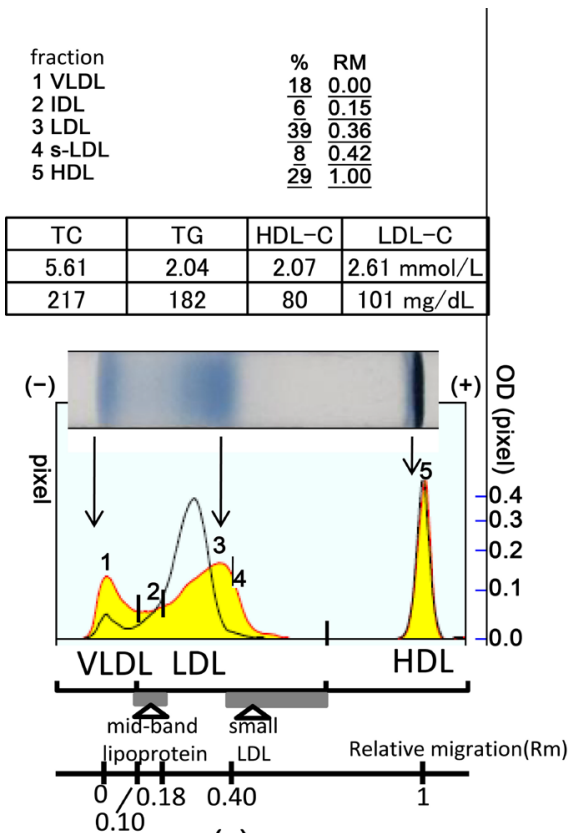

(a)

\begin{tabular}{|c|c|c|}
\hline $\begin{array}{l}\text { fraction } \\
1 \text { VLDL } \\
2 \text { IDL } \\
3 \text { LDL } \\
4 \text { s-LDL } \\
5 \text { HDL }\end{array}$ & $\begin{array}{l}\% \\
\frac{40}{3} \\
\frac{3}{9} \\
\frac{2}{46}\end{array}$ & $\begin{array}{l}\text { RN } \\
0.0 \\
0.1 \\
0.3 \\
0.4 \\
1.0\end{array}$ \\
\hline
\end{tabular}

\begin{tabular}{|c|c|c|c|}
\hline TC & TG & HDL-C & LDL-C \\
\hline 3.47 & 3.53 & 1.76 & $0.08 \mathrm{mmol} / \mathrm{L}$ \\
\hline 134 & 315 & 68 & $3 \mathrm{mg} / \mathrm{dL}$ \\
\hline
\end{tabular}

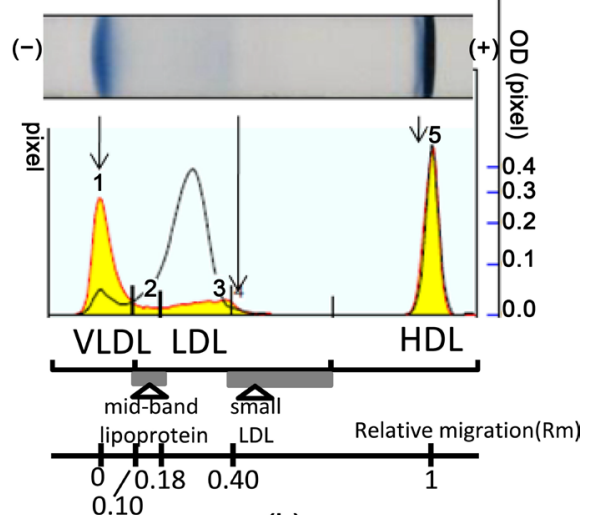

(b)

Figure 2. Case in which LDL-cholesterol disappears by short-term treatment with evolocumab. We indicated an example of the lipoprotein densitometric patterns by $3 \%$ PAGE before (a) and after (b) the add-on therapy of $140 \mathrm{mg}$ evolocumab. Although there were marked changes in the lipid profiles, the changes of the mean LDL sizes were not big, "Quantity" and "quality" of lipoproteins seem to be clinically valuable. The solid line shows the reference densitometric pattern. The sample densitometric pattern of a patient (yellow filled areas) was overlaid with the reference pattern. The pattern was separated into 3 fractions: VLDL, LDL, and HDL. The area between Rm 0.10 and Rm 0.18 was assigned as mid-band lipoproteins. In addition, the excess area on the right side of the LDL peak $(\mathrm{Rm}>0.40)$ was assigned as small LDL. The levels of lbLDL-C were calculated by subtracting sdLDL-C and mid-band lipoprotein-C levels from total LDL-C. This pattern after (b) the add-on therapy of $140 \mathrm{mg}$ evolocumab was similar to those with Type $\mathrm{V}$ hyperlipidemia. The LDL-cholesterol disappears by short-term treatment with evolocumab. Abbreviations: LDL, low-density lipoprotein; lbLDL, large buoyant LDL; sdLDL, small dense LDL.

were $27.6 \pm 0.6 \mathrm{~nm}, 18.9 \pm 0.3 \mathrm{~nm}, 16.3 \pm 0.4 \mathrm{~nm}$, and $13.6 \pm 1.0 \mathrm{~nm}$, respectively (Figure 4(a), Figure 4(b)). The VLDL size marker was made using a $30 \mathrm{~nm}$ gold colloid standard sample (Reference Material ${ }^{\circledR} 8012$, National Institute of Standards $\&$ Technology). The diameters of the size markers measured by transmission electron microscope were smaller than those determined by DLS. The data obtained by DLS as a result of cumulant calculation indicated that the diameters of the size markers exhibited in the order of IDL marker > LDL marker > small LDL marker at an interval of about $2 \mathrm{~nm}$, which agrees with the data obtained by the transmission electron microscope.

\subsection{Changes in the Mean LDL Size by the Add-On Evolocumab Therapy and Relationships among the Main LDL Particle Diameters Obtained with 2\% - 16\% GGE, 2.7\% - 5\% GGE, and $3 \%$ PAGE}

We indicated the electrophoresis images of three size markers (Figures 5(a)-(c)). 
Moreover, we indicated the standard curve obtained from the relationship between the diameters of the size markers and the Rm values in $2 \%-16 \%$ GGE (data not shown), 2.7\% - 5\% GGE (data not shown), and 3\% PAGE (Figure 6). In patients before and two and four weeks after initiation of the evolocumab treatment, the relationships between the mean LDL particle diameters obtained with $2 \%$ - 16\% GGE, 2.7\% - 5\% GGE, and 3\% PAGE were analyzed (Figure 7). The quantities obtained by the conventional, verified method using $2 \%-16 \%$

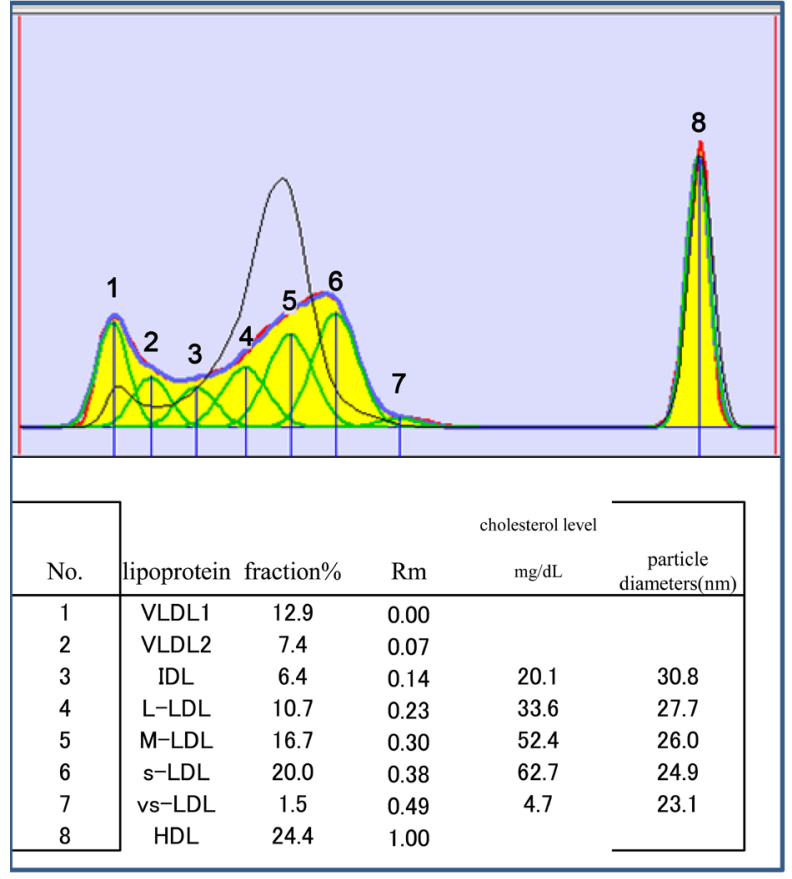

(a)

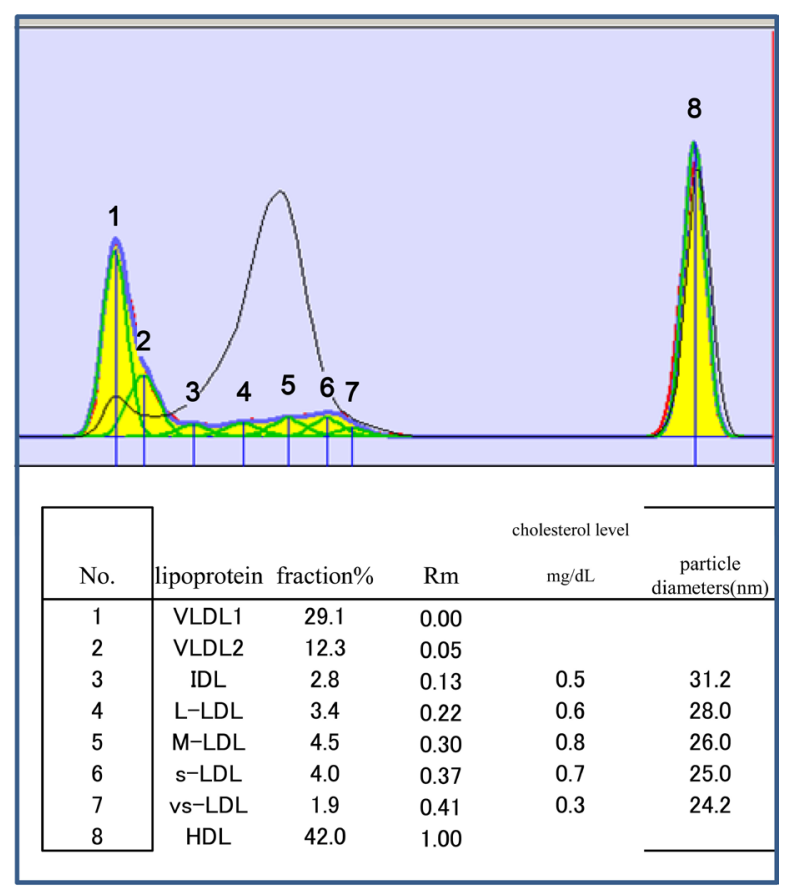

(b)

Figure 3. Evaluation of changes of Figure 2 in the eight fractions before (a) and after (b) the add-on therapy of $140 \mathrm{mg}$ evolocumab by the Gaussian fitting method. We indicated an example of the lipoprotein Gaussian fitting patterns. Short-term evolocumab treatment reduced plasma s-LDL plus vs-LDL level. By this Gaussian fitting method, eight fractions can be detected: VLDL1, VLDL2, IDL, L-LDL, M-LDL, s-LDL, vs-LDL, and HDL fractions. The IDL fraction consistent with mid-band lipoprotein particle. The L-LDL and M-LDL fractions correspond to lbLDL particles, whereas the s-LDL and vs-LDL fractions correspond to sdLDL particles. Abbreviations: VLDL, very low-density lipoprotein; IDL, intermediate-density lipoprotein; L-LDL, large size LDL; M-LDL, medium size LDL; s-LDL, small size LDL; vs-LDL, very small size LDL; lbLDL, large buoyant LDL; sdLDL, small dense LDL.

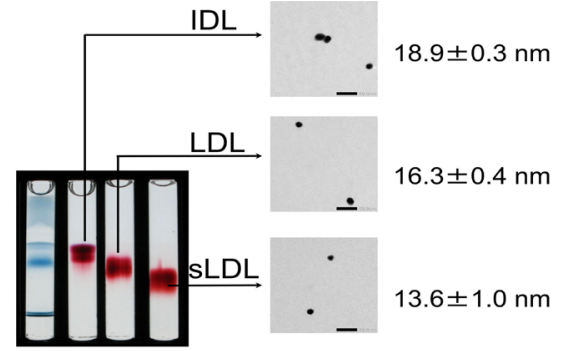

(a)

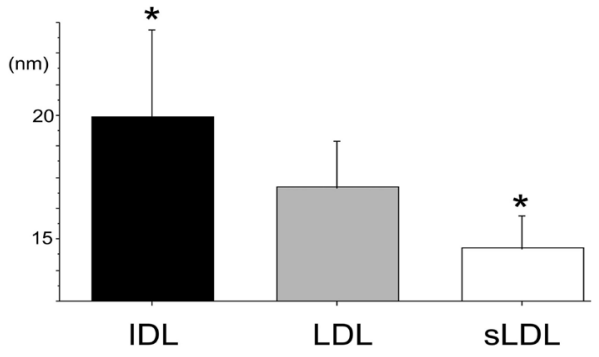

(b)

Figure 4. Albumin-bound gold nanoparticles maked by new method of constructing artificial lipoprotein size markers. (a) Albumin-bound gold nanoparticles were subjected by PAGE. Each fraction was observed by transmission electron microscope. The diameters of the gold nanoparticles were $18.9 \pm 0.3,16.3 \pm 0.4,13.6 \pm 1.0 \mathrm{~nm}$, respectively. Scale bar $=50 \mathrm{~nm}$. (b) Size distribution of gold nanoparticles of each fraction. Values are the mean \pm standard deviation. ${ }^{*}$ significantly different from LDL size marker $(\mathrm{p}$ $<0.001$, by paired- $t$ test). Black box, IDL size marker; gray box, LDL size marker; white box, small LDL size marker. 


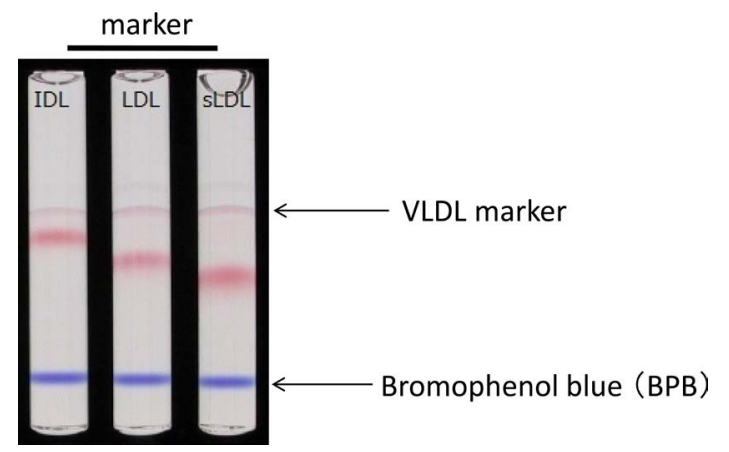

(a)

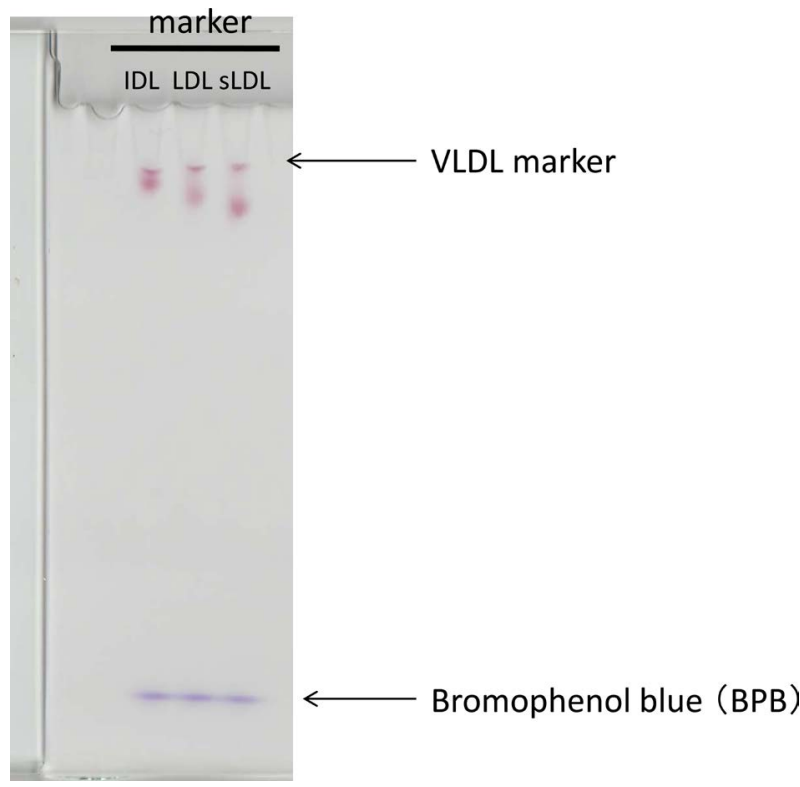

(b)

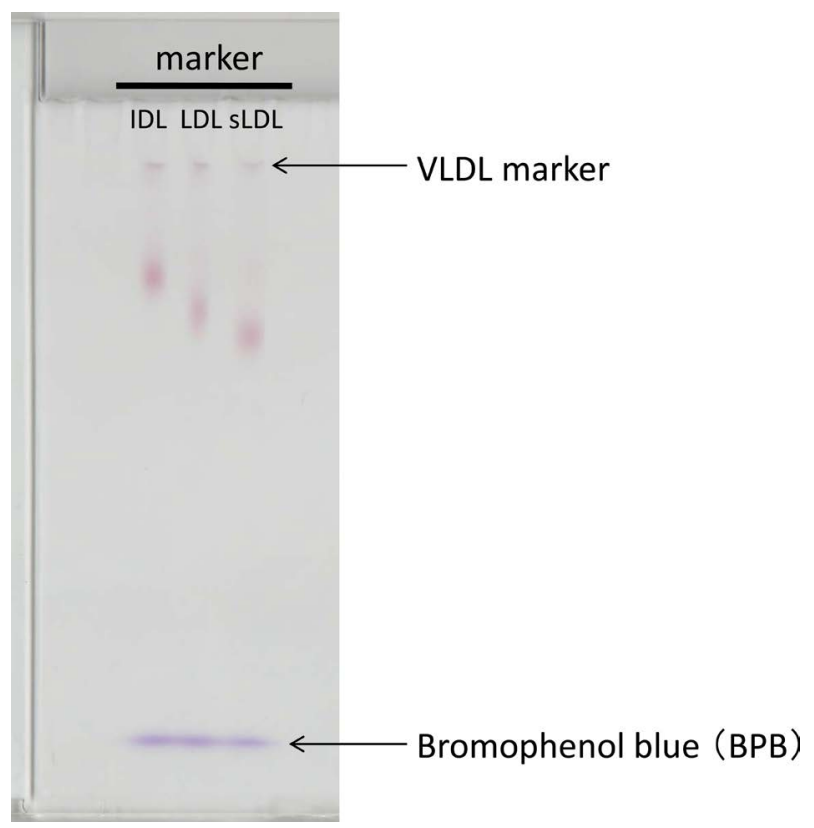

(c)

Figure 5. Representative images of 3\% PAGE (a), 2\% - 16\% GGE (b) and 2.7\% - 5\% GGE (c) along with electrophoresis images of three size markers. 


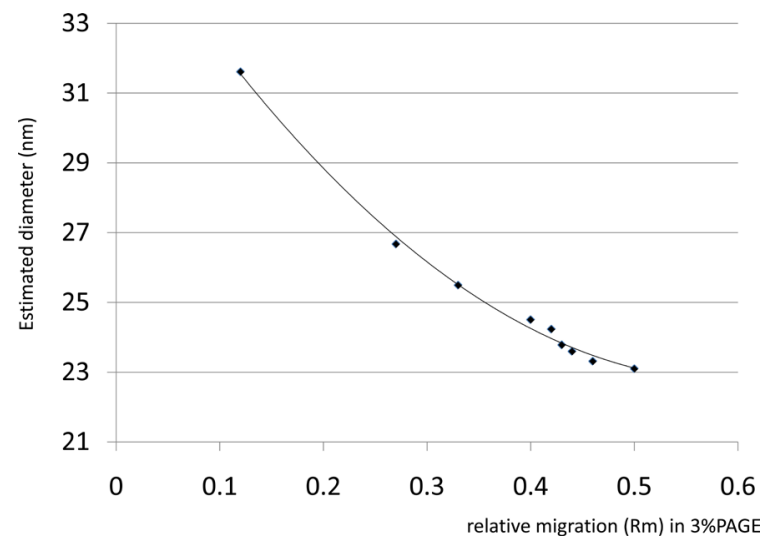

Figure 6. The standard curve obtained from the relationship between the diameters $(\mathrm{nm})$ of the size markers and the relative migration $(\mathrm{Rm})$ values for 3\% PAGE. We make the various diameters of the size markers and estimated Rm values in 3\% PAGE.
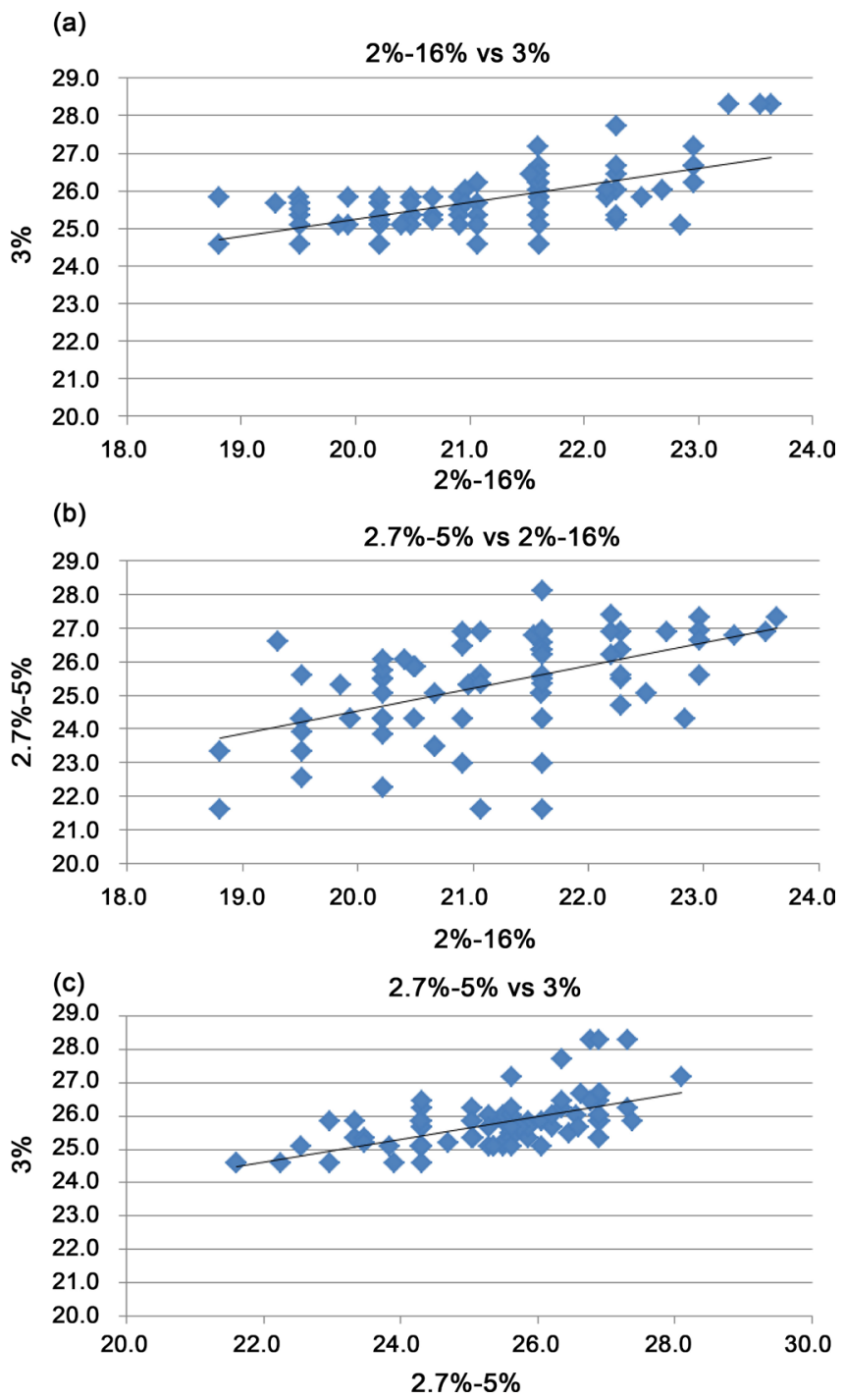

Figure 7. Relationships among the main LDL particle diameters (nm) obtained with $2 \%$ - 16\% GGE, $2.7 \%-5 \%$ GGE, and $3 \%$ PAGE. (a) The data obtained from the $2 \%-16 \%$ GGE significantly correlated with those from the 3\% PAGE (correlation coefficient $\mathrm{R}=0.621, \mathrm{p}<0.05$ ). (b) The data obtained from the $2 \%-16 \%$ GGE significantly correlated with those from the $2.7 \%-5 \%$ GGE (correlation coefficient $\mathrm{R}=0.516, \mathrm{p}<0.05$ ). (c) There was also a good correlation between the $3 \%$ PAGE and the $2.7 \%-5 \%$ GGE (correlation coefficient $\mathrm{R}=0.612, \mathrm{p}<0.05$ ). 
GGE were compared with those obtained by 3\% PAGE or 2.7\% - 5\% GGE, which showed that there were correlations between the former and the latter data (3\% PAGE: correlation coefficient $\mathrm{R}=0.621, \mathrm{p}=6.0 \times 10^{-10} ; 2.7 \%-5 \%$ GGE: correlation coefficient $\mathrm{R}=0.516, \mathrm{p}=8.4 \times 10^{-7}$ ); there was also a good correlation between 3\% PAGE and 2.7\% - 5\% GGE (correlation coefficient R $=0.612$, p $\left.=1.3 \times 10^{-9}\right)$.

With the add-on evolocumab therapy, the mean LDL size obtained with $2 \%$ $16 \%$ GGE was significantly increased in the 30 patients from $20.8 \pm 0.9 \mathrm{~nm}$ to $22.6 \pm 1.7 \mathrm{~nm}$. Changes in the main LDL particle diameter obtained with $2.7 \%-$ $5 \%$ GGE from $24.8 \pm 1.2 \mathrm{~nm}$ to $27.2 \pm 2.3 \mathrm{~nm}$ and $3 \%$ PAGE were significantly increased from $25.6 \pm 0.4 \mathrm{~nm}$ to $26.4 \pm 0.8 \mathrm{~nm}$ (Figure 8 ).

\subsection{Changes in the LDL Subfraction Due to the Add-On Evolocumab Therapy as Determined by 3\% PAGE (LipoPhor AS ${ }^{\circledR}$ )}

Figure 9(a) shows the sdLDL-C, lbLDL-C, and mid-band lipoprotein-C-lowering effect of subcutaneous doses of evolocumab administered every 2 weeks. The sdLDL-C, lbLDL-C, and mid-band lipoprotein-C levels were respectively reduced by $90 \%, 47 \%$, and $36 \%$. Figure 9 (b) shows the changes to the sdLDL-C/total LDL-C ratio, lbLDL-C/total LDL-C ratio, mid-band lipoprotein-C/total LDL-C ratio, and sdLDL-C/lbLDL-C ratio due to administration of $140 \mathrm{mg}$ evolocumab every two weeks. The sdLDL-C/total LDL-C ratio, lbLDL-C/total LDL-C ratio, and sdLDL-C/lbLDL-C ratio were improved after evolocumab treatment respectively, while the mid-band lipoprotein-C/total LDL-C ratio were not improved (Figure 9(b)).

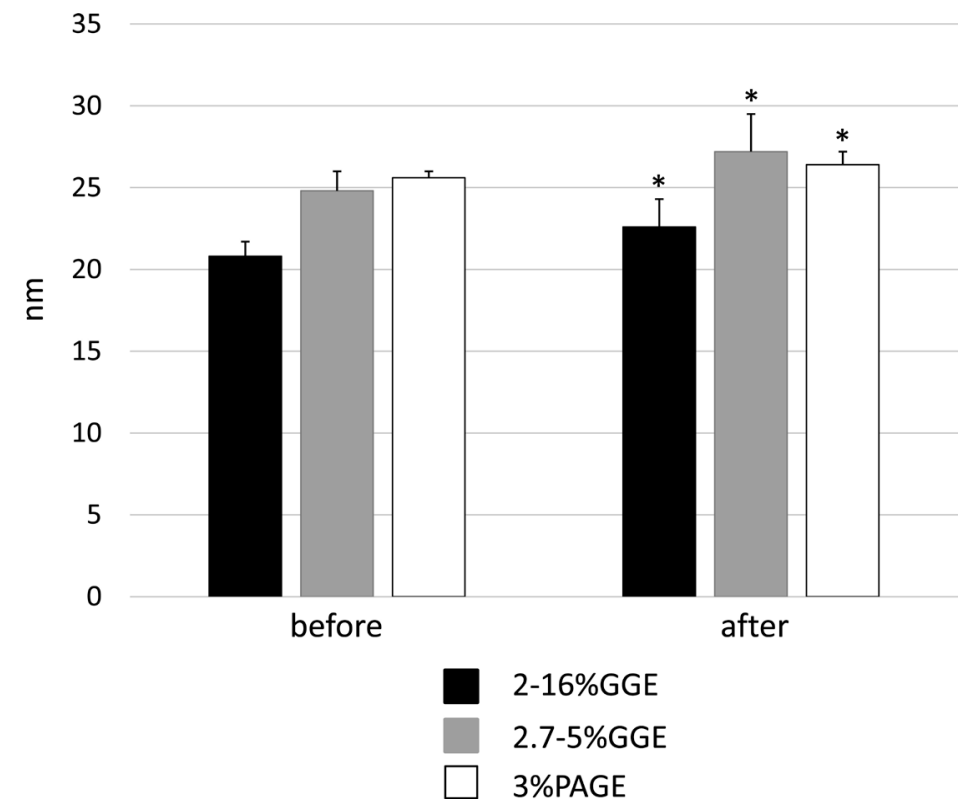

Figure 8. Changes in the mean LDL size before and after the add-on therapy of $140 \mathrm{mg}$ evolocumab $(n=30)$. All values are mean \pm standard deviations. * significantly different from values obtained before evolocumab therapy ( $\mathrm{p}<0.001$, by paired- $t$ test). With the add-on evolocumab therapy, the mean LDL size obtained with the poly-acrylamide (gradient) gel electrophoresis methods (GGE and PAGE) was significantly increased. 


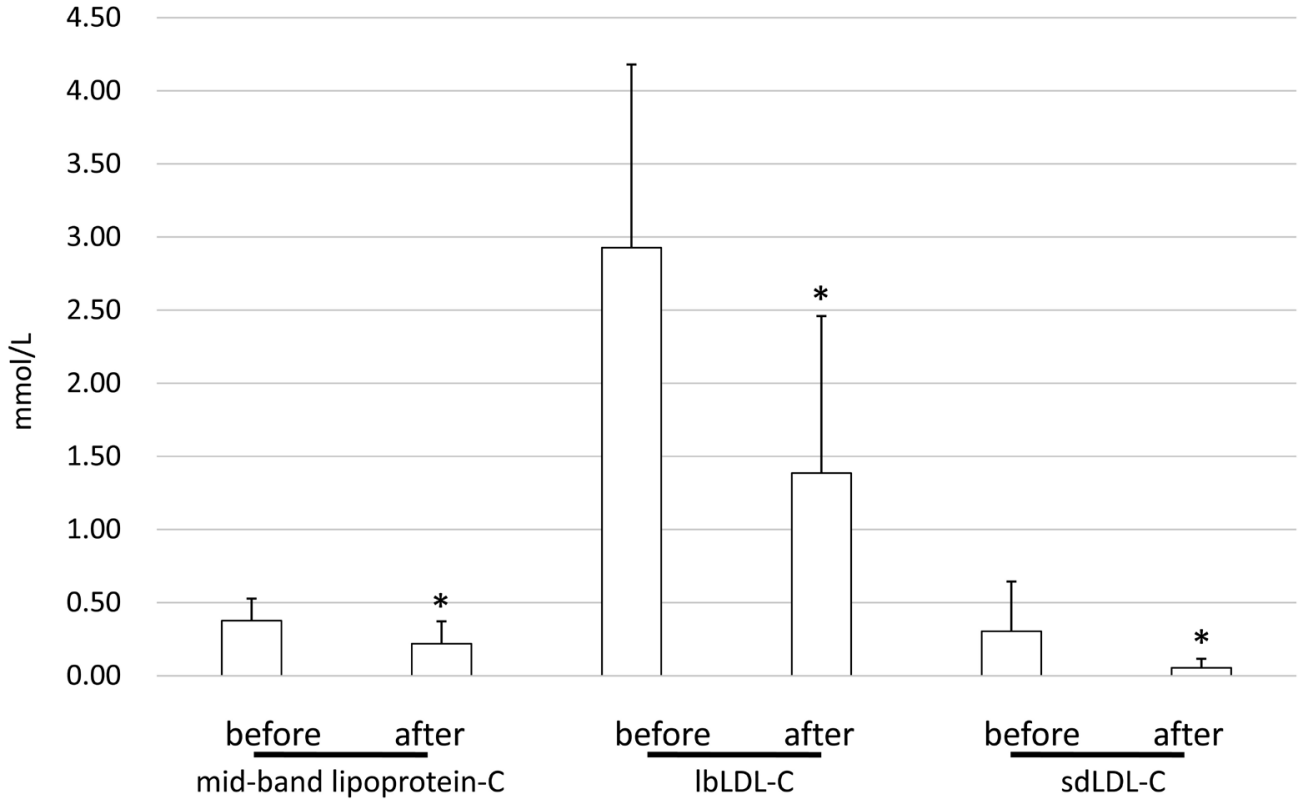

(a)

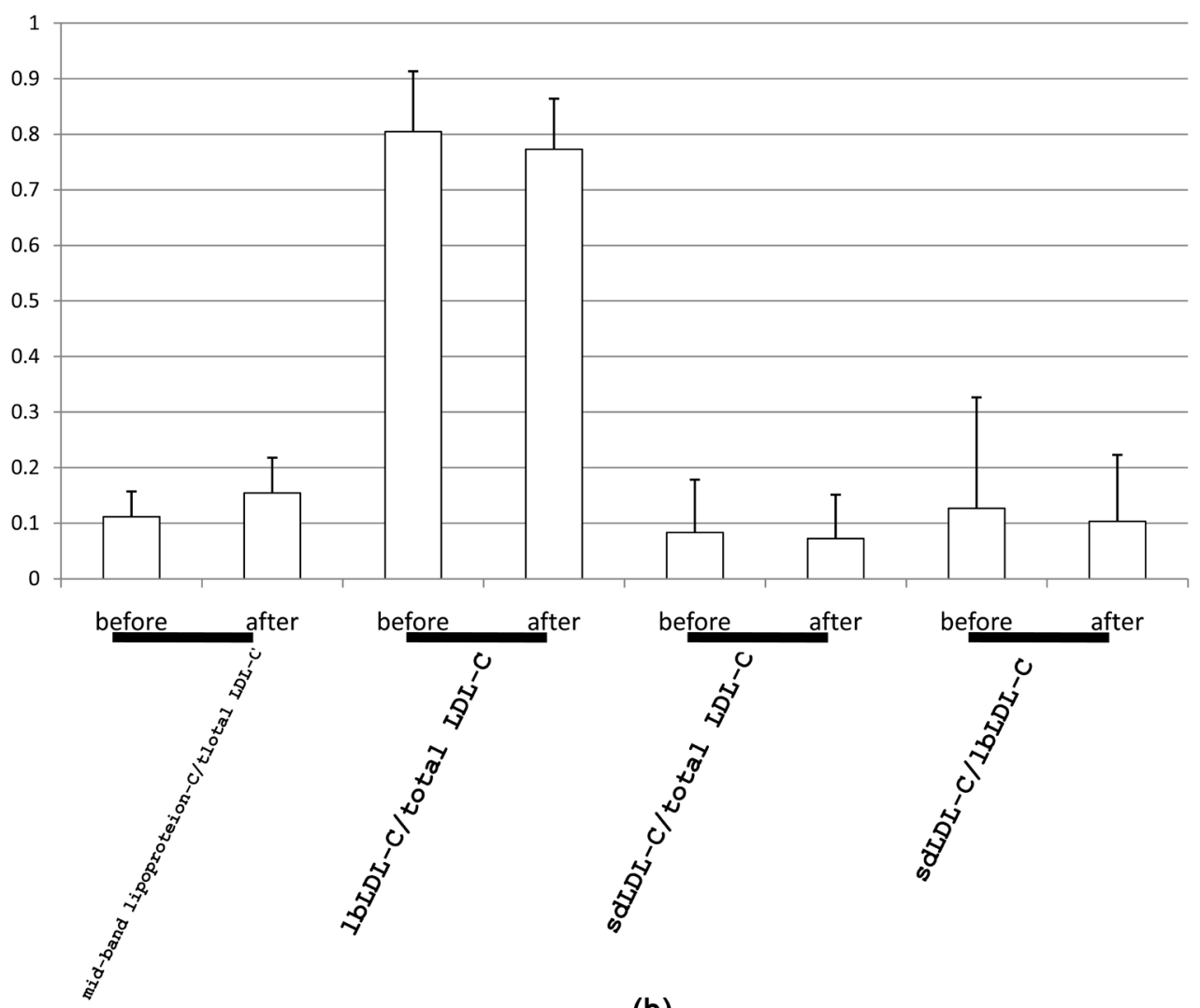

(b)

Figure 9. (a) Change of LDL-C, lipoprotein LDL-C, lbLDL-C, and sdLDL-C levels and (b) change of the sdLDL-C/total LDL-C ratio, lbLDL-C/total LDL-C ratio, mid-band lipoprotein-C/total LDL-C ratio, and sdLDL-C/lbLDL-C ratio before and after the add-on therapy of $140 \mathrm{mg}$ evolocumab $(\mathrm{n}=30)$. The sdLDL-C, lbLDL-C, and mid-band lipoprotein-C levels were respectively reduced by $90 \%, 47 \%$, and $36 \%$. The sdLDL-C/total LDL-C ratio, lbLDL-C/total LDL-C ratio, and sdLDL-C/lbLDL-C ratio were improved after evolocumab treatment, respectively, while the mid-band lipoprotein-C/total LDL-C ratio were not improved. All values are mean \pm standard deviations. Abbreviations: LDL, low-density lipoprotein; lbLDL, large buoyant LDL; sdLDL, small dense LDL. 


\section{Discussion}

Our data (Figure 8) obtained from 2\% - 16\% GGE demonstrated that the shortterm evolocumab treatment increased the mean LDL size significantly and dramatically. The $2 \%-16 \%$ GGE is regarded as the golden standard method for LDL subfraction analysis or for estimation of LDL particle size [5] [14]. By using LDL size maker (Figures 5(a)-(c)), we indicated that the main LDL particle size estimated by lipoprotein migration in 2\% - 16\% GGE has been shown to be highly correlated with those obtained by 3\% PAGE and $2.7 \%-5 \%$ GGE (Figure 7). The 3\% PAGE also demonstrated that evolocumab treatment significantly reduced the sdLDL-C, lbLDL-C, and mid-band lipoproteins-C (Figure 9(a)). Overlaying the PAGE results onto a standard densitometry pattern enabled us to identify the presence of sdLDL and/or mid-band lipoproteins (Figure 2). Moreover, the use of $\mathrm{Rm}>0.40$ for an LDL peak estimated sdLDL content with sufficient accuracy (Figure 2). The results obtained from 3\% PAGE were completely consistent with those obtained by $2 \%-16 \%$ GGE and $2.7 \%-5 \%$ GGE.

Mechanisms for the generation of smaller-sized LDL have been proposed [24] [25]. There is substantial evidence that type 2 diabetes mellitus, insulin resistance, overeating, and/or obesity may increase free fatty acid release into circulation from adipocytes and elevate plasma glucose [23]. The increased free fatty acids and plasma glucose are utilized as substrates for hepatic TG synthesis. As a consequence of increased hepatic TG synthesis, there may be increased production of TG-rich (large-sized) VLDL in the liver. This TG-rich (large-sized) VLDL can be a precursor of sdLDL. TG-rich (large-sized) VLDL is converted to TGenriched LDL, the favored substrate for hepatic lipase, which is transformed into smaller-sized LDL by lipase-mediated TG hydrolysis [26]. In fact, as data not shown, our data indicated that evolocumab treatment significantly reduced the AUC (\%) for VLDL and the concentration (mg/dL) of VLDL1, which is larger than VLDL2 (Figure 10). Evolocumab may reduce plasma TG levels via prevention of VLDL assembly. It is interesting that PCSK9 may be related to VLDL production in the liver. Recently, it has been reported that PCSK9 is associated with the clearance of VLDL receptors, as well as LDL receptors [27], in the liver. In addition, the effect of PCSK9 on intestinal TG-rich lipoprotein production has been reported [28]. Further research is needed to resolve this problem.

Our data presented that TG levels, as well as TC and LDL-C levels, were also improved after four-week evolocumab treatments with the administration of 140 $\mathrm{mg} /$ day as an add-on therapy to statin (Figure 1). TG level was associated with level of lipoprotein; sdLDL, IDL, TG rich lipoprotein (TRL). It had been reported that sdLDL was powerfully associated with TG levels [29] [30]. The sdLDL-C level was significantly reduced with the administration of $140 \mathrm{mg} /$ day of evolocumab as an add-on therapy to statin, resulting in possible TG level reduction. Mid-band lipoproteins detected in the area between the VLDL and LDL bands by PAGE mainly include IDL, lipoprotein (a) (Lp(a)) [31], and TRL remnants [32]. Our data indicated that the mean mid-band lipoprotein particle di- 


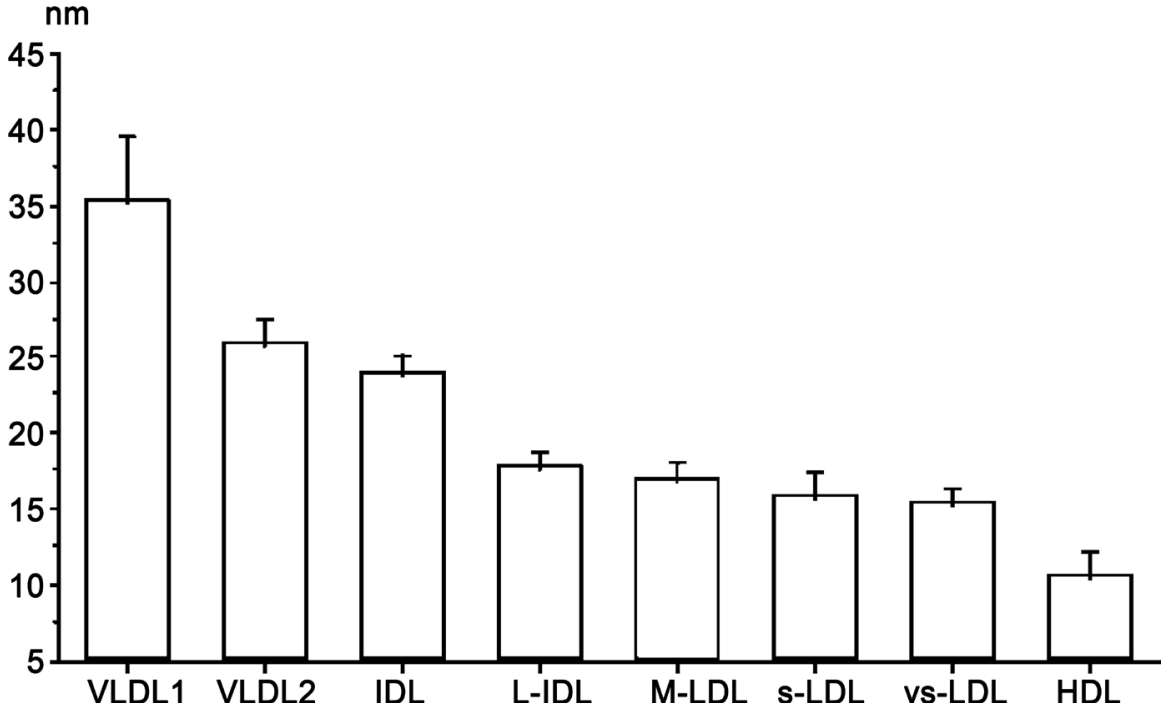

Figure 10. Size of the extracted VLDL1, VLDL2, IDL, L-LDL, M-LDL, s-LDL, vs-LDL and HDL particles. Size distribution of the extracted lipoprotein particles were examined by transmission electron microscope. The representative result indicated that the main diameters (nm) of VLDL1, VLDL2, IDL, L-LDL, M-LDL, s-LDL, vs-LDL, and HDL were $35.4,25.9,23.9,17.9,17.0,15.9,15.4$, and 10.6, respectively. Values are the mean \pm standard deviation. Abbreviations: VLDL, very low-density lipoprotein; IDL, intermediatedensity lipoprotein; L-LDL, large size LDL; M-LDL, medium size LDL; s-LDL, small size LDL; vs-LDL, very small size LDL.

ameter was 32.2 (Figure 11) nm, VLDL2 and IDL particle diameter was about 25 (Figure 10) nm, which were determined by the Phoreto-yield method [33] [34]. This mid-band lipoprotein particle was TRL and/or IDL, resulting in possible TG level reduction. In fact, the administration of evolocumab decreased cholesterol content in the mid-band lipoprotein fraction (Figure 9(a)), which indicated that evolocumab may have the power to reduce the level of IDL and TRL remnant lipoproteins. TRL remnants were also easily assayed for by use of two commercial kits, Metabo Lead RemC [35] (Kyowa Medex, Tokyo) [36] and RLPcholesterol JIMRO-II (Otsuka Pharmaceutica1 Co, Ltd., Kagawa, Japan). The values given by the MetaboLead RemC kit or by the RLP-cholesterol kit may be used for further analysis.

Our data presented that mid-band lipoprotein fraction levels were also reduced with evolocumab treatments (Figure 9(a)). As above mentioned, midband lipoproteins include lipoprotein (a) ( $\mathrm{Lp}(\mathrm{a})$ ) [31] in addition to IDL and TRL remnants. Recently, it has been reported that evolocumab treatment decreases $\mathrm{Lp}$ (a) levels. In vivo and in vitro data in this analysis support the hypothesis that the additional upregulation of LDLR activity by PCSK9 mAb also increases the clearance of $L p(a)$ [37].

Today, statins are frequently clinically used as the drugs of first choice for the treatment of hyperlipidemia. These drugs have a strong TC and LDL-C reducing effect, and their safety has been evaluated highly. A number of patients who are treated relatively frequently by general physicians are multiple risk patients who have coronary risk factors, such as hypertension and diabetes mellitus, and the 

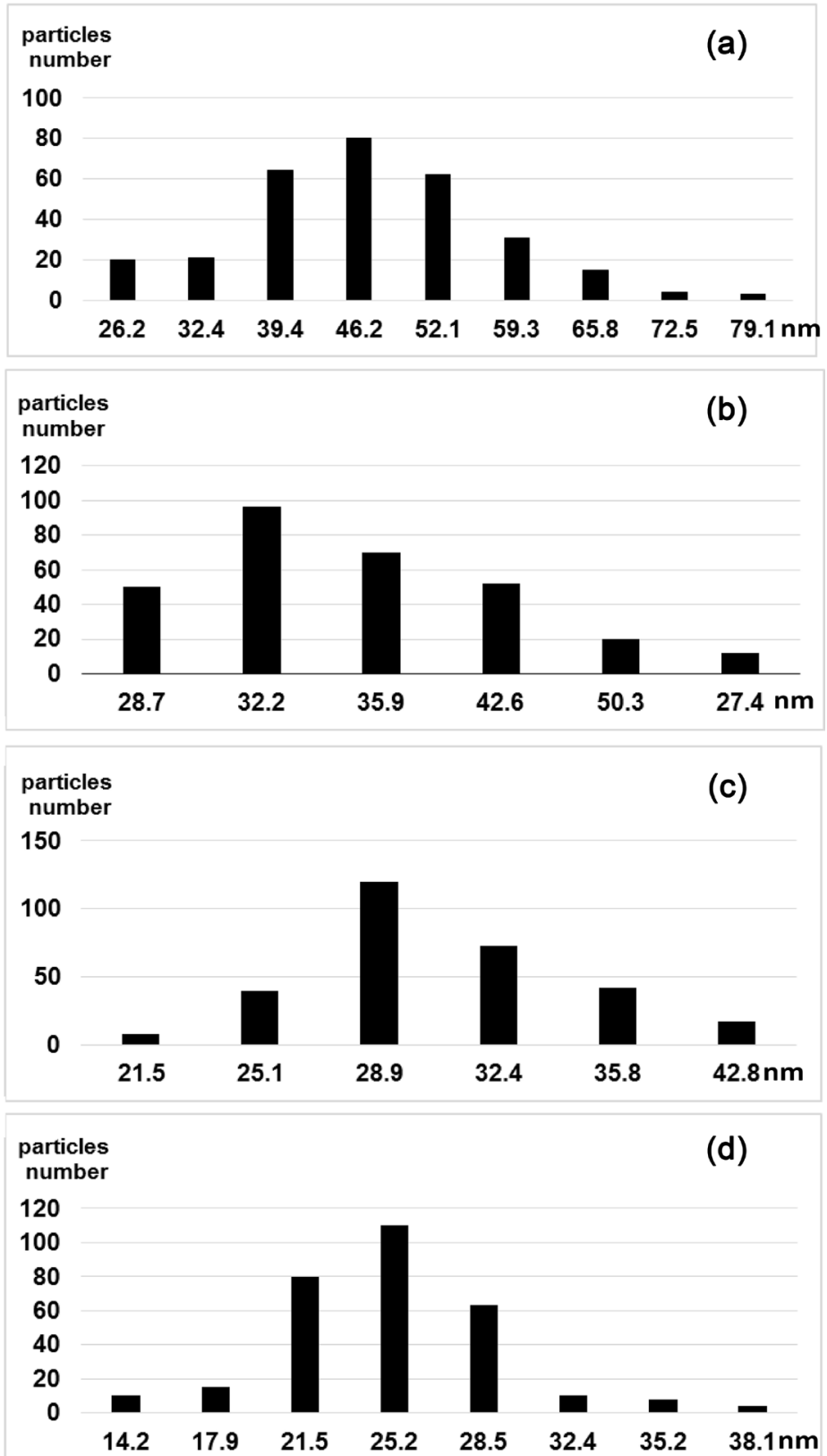

Figure 11. Size distribution of the extracted VLDL (a), mid-band (b), lbLDL (c), sdLDL (d) particles number. We recovered and examined the lipoprotein particles from each gel-block that contained lipoproteins after electrophoresis by 3\% PAGE using the transmission electron microscope in order to determine the diameter of VLDL, mid-band lipoproteins, lbLDL, sdLDL particles. The representative results indicated that the main diameters $(\mathrm{nm})$ of VLDL, mid-band lipoproteins, lbLDL, and sdLDL particles were 46.2, $32.2,28.9$, and 25.2 , respectively.

ordinary dosage is sometimes insufficient for patients with moderate to severe hypercholesterolemia. In the "2012 Guidelines for Treatment of Dyslipidemia" by the JAS, risk factors besides hyperlipidemia are also carefully considered, and emphasis is placed on understanding the multiple risks resulting from the accumulation of such risks. In actual treatment, the target lipid control level is set up in consideration of the presence or absence of CAD, the number of major 
coronary risks, etc., and individually tailor-made control and reduction of risks should be set up as the goal. We called it the treatment to "optimal" target, moreover, we proposed the concept that there was target level that could completely prevent cardiovascular events. We called it the treatment to "ZERO" target (under preparation). LDL-C level for diabetes mellitus with acute coronary syndrome was less than $50 \mathrm{mg} / \mathrm{dL}$, secondary prevention was less than $60 \mathrm{mg} / \mathrm{dL}$, primary prevention was less than $70 \mathrm{mg} / \mathrm{dL}$, respectively.

In the present study, we selected 30 patients with dyslipidemia being treated with statins who were in the high-risk group and administered evolocumab at a dosage of $140 \mathrm{mg}$ every two weeks. In these patients, the mean TC level before the administration of evolocumab was $5.772 \mathrm{mmol} / \mathrm{L}(223.2 \mathrm{mg} / \mathrm{dL})$, and mean LDL-C was $3.543 \mathrm{mmol} / \mathrm{L}(137.0 \mathrm{mg} / \mathrm{dL})$. It was possible by use of evolocumab to reduce the mean LDL-C to the target control value for the high-risk group and $<80 \mathrm{mg} / \mathrm{dL}$ in the secondary prevention group, resulting in a goal attainment rate of $100 \%$. In past studies on the evolocumab add-on therapy in patients who did not show sufficient decrease in lipid level under statin therapy, additional reducing effects were observed in percent changes in LDL-C (-75\%) [38]. In our present short-term study, to the same extent, TC and LDL-C were significantly reduced, showing the effect of evolocumab therapy (Figure 1).

However, there are many cases in which LDL-C disappear after two weeks of evolocumab treatment (Figure 2(b)). The Phase-III trials with $\mathrm{mAb}$ inhibiting PCSK9 exhibited memory-loss and confusion in $1 \%-1.5 \%$ of the study subjects [39] indicating that lipid lowering through PCSK9 inhibition requires careful therapeutic monitoring. We propose the administration every $3 \sim 4$ weeks of 140 $\mathrm{mg}$ /day of evolocumab as an add-on to statin therapy after lipoprotein particles are monitored by $3 \%$ PAGE. Moreover, the apoprotein E (APOE) $\varepsilon 4$ allele is a known genetic risk factor for $\mathrm{AD}$ by accelerating the onset of $\mathrm{AD}$ [40], whereas APOE $\varepsilon 2$ is protective [41]. It may be important to look at setting up a goal for the reduction of LDL-C by evolocumab treatment according to APOE phenotype in addition to estimating the risk factor for atherosclerosis.

Further investigations, such as large randomized trials with long follow-up periods, are needed to determine whether it is possible to prevent CAD by using evolocumab therapy to change the mean LDL size. In our study, cardiac computed tomography (CT) angiography of the coronary arteries has been performed in some patients who participated in this clinical study. The cardiac CT angiography of the coronary arteries will be performed after a six-month follow-up period in patients who participated in this clinical study.

\section{Limitations}

The present study had several limitations. First, the enrolled number of subjects was markedly small, and this study had an open-label design.

Second, the markedly short study duration of four weeks may also be a limitation. However, since evolocumab is known to act rapidly, the evaluation of evolocumab treatment after four weeks may be sufficient to observe the maximal li- 
pid effect of the study. On the other hand, there was a possibility that the treatment period was probably too short to obtain a full therapeutic effect. Therefore, a long-term, larger-scale, and double-blind study in which the drugs are administered to patients with high-risk hyperlipidemias is needed in the future.

Third, a selection bias could be present because most patients were selected based on the detection of small size LDL-C after receiving $5 \mathrm{mg}$ to $20 \mathrm{mg}$ rosuvastatin or $10 \mathrm{mg}$ to $40 \mathrm{mg}$ atorvastatin for more than 24 weeks. Therefore, it is possible that patients for whom rosuvastatin or atorvastatin were less effective in lowering small size LDL-C levels were selected at screening. We should not ignore the fact that many patients treated with rosuvastatin or atorvastatin would be expected to have a good response in terms of the small size LDL-C level. Using a long-term study design, it should be investigated whether CAD can or cannot be prevented by lowering small size LDL-C by using evolocumab as an add-on to statin therapy.

Fourth, there may be differences between the genotype and phenotype when the apolipoprotein $\mathrm{E}$ phenotypes were assessed.

Fifth, we cannot completely eliminate the effects of LDL particle charge as this system uses $3 \%$ pre-cast polyacrylamide gels.

Finally, we excluded subjects with TG levels $>4.5 \mathrm{mmol} / \mathrm{L}$ ( $400 \mathrm{mg} / \mathrm{dL})$, because we calculated the LDL-C level using the Friedewald equation. Also, all the participants were Japanese; therefore, our findings cannot be generalized to other races or ethnic groups.

\section{Conclusion}

Our present study suggests that evolocumab every two weeks as an add-on to statin therapy may be a useful therapeutic option to reduce small size LDL-C levels in Japanese hypercholesterolemic patients with high-risk hyperlipidemias after long-term statin treatment.

\section{Acknowledgements}

The authors thank Sawako Satoh for technical and secretarial assistance.

\section{Conflict of Interest}

No conflict of interest declared.

\section{Financial Support}

This work was supported by JSPS KAKENHI Grant Number 26461367 and by a Grant-In-Aid from Saitama Industrial Promotion Public Corporation.

\section{References}

[1] Mudd, J.O., Bortaug, B.A., Johnston, P.V., Kral, B.G., Rouf, R., Blumenthal, R.S. and Kwiterovich, P.O. (2007) Beyond Low-Density Lipoprotein Cholesterol Defining the Role of Low-Density Lipoprotein Heterogeneity in Coronary Artery Disease. American College of Cardiology, 50, 1735-1741. https://doi.org/10.1016/j.jacc.2007.07.045 
[2] Inoue, I. and Katayama, S. (2004) The Possible Therapeutic Actions of Peroxisome Proliferator-Activated Receptor Alpha (PPAR Alpha) Agonists, PPAR Gamma Agonists, 3-Hydroxy-3-Methylglutaryl Coenzyme A (HMG-CoA) Reductase Inhibitors, Angiotensin Converting Enzyme (ACE) Inhibitors and Calcium (Ca)-Antagonists on Vascular Endothelial Cells. Current Drug Targets-Cardiovascular \& Hematological Disorders, 4, 35-52. https://doi.org/10.2174/1568006043481329

[3] Krähenbühl, S., Pavik-Mezzour, I. and von Eckardstein, A. (2016) Unmet Needs in LDL-C Lowering: When Statins Won't Do! Drugs, 76, 1175-1190.

https://doi.org/10.1007/s40265-016-0613-0

[4] Havel, R.J. (2000) Remnant Lipoproteins as Therapeutic Targets. Current Opinion in Lipidology, 11, 615-620. https://doi.org/10.1097/00041433-200012000-00008

[5] Chapman, M.J., Guerin, M. and Bruckert, E. (1998) Atherogenic, Dense Low-Density Lipoproteins. Pathophysiology and New Therapeutic Approaches. European Heart Journal, 19, A24-A30.

[6] Nichols, A.V., Krauss, R.M. and Musliner, T.A. (1986) Nondenaturing Polyacrylamide Gradient Gel Electrophoresis. Methods in Enzymology, 128, 417-431.

https://doi.org/10.1016/0076-6879(86)28084-2

[7] Austin, M.A., Breslosv, J.L., Hennekens, C.H., Buring, J.E., Willett, W.C. and Krauss, R.M. (1988) Low-Density Lipoprotein Subclass Patterns and Risk of Myocardial Infarction. JAMA, 260, 1917-1921. https://doi.org/10.1001/jama.1988.03410130125037

[8] Nozue, T., Michishita, I., Ito, Y. and Hirano, T. (2008) Effects of Statin on Small Dense Low Density-Lipoprotein Cholesterol and Remnant-Like Particle Cholesterol in Heterozygous Hypercholesterolemia. Journal of Atherosclerosis and Thrombosis, 15, 146-153. https://doi.org/10.5551/jat.E552

[9] Inoue, I., Awata, T. and Katayama, S. (2010) Retrospective, Observation Study: Quantitative and Qualitative Effect of Ezetimibe and HMG-CoA Reductase Inhibitors on LDL-Cholesterol: Are There Disappearance Thresholds for Small, Dense LDL and IDL? Recent Patents on Cardiovascular Drug Discovery, 5, 143-152. https://doi.org/10.2174/157489010791515386

[10] Lambert, G., Sjouke, B., Choque, B., Kastelein, J.J. and Hovingh, G.K. (2012) The PCSK9 Decade. Journal of Lipid Research, 53, 2515-2524.

https://doi.org/10.1194/jlr.R026658

[11] Nakano, T., Inoue, I., Seo, M., Takahashi, S., Awata, T., Komoda, T. and Katayama, S. (2009) Rapid and Simple Profiling of Lipoproteins by Polyacrylamide-Gel Disc Electrophoresis to Determine the Heterogeneity of Low-Density Lipoproteins (LDLs) Including Small, Dense LDL. Recent Patents on Cardiovascular Drug Discovery, 4, 31-36. https://doi.org/10.2174/157489009787260034

[12] O’Neal, D., Harrip, P., Dragicevic, G., Rae, D. and Best, J.D. (1998) A Comparison of LDL Size Determination Using Gradient Gel Electrophoresis and Light-Scattering Methods. Journal of Lipid Research, 9, 2086-2090. http://www.jlr.org/content/39/10/2086.long

[13] Hirayama, S. and Miida, T. (2012) Small Dense LDL: An Emerging Risk Factor for Cardiovascular Disease. Clinica Chimica Acta, 414, 215-224.

https://doi.org/10.1016/j.cca.2012.09.010

[14] Ensign, W., Hill, N. and Heward, C.B. (2006) Disparate LDL Phenotypic Classification among 4 Different Methods Assessing LDL Particle Characteristics. Clinical Chemistry, 52, L722-L727. https://doi.org/10.1373/clinchem.2005.059949

[15] Ikeda, T., Seo, M., Inoue, I., Katayama, S., Matsunaga, T., Hara, A., Komoda, T. and Tabuchi, M. (2010) Direct and Simple Fluorescence Detection Method for Oxidized 
Lipoproteins. Analytical Chemistry, 82, 1128-1132. https://doi.org/10.1021/ac902018a

[16] Stein, E.A. (2006) Are Measurements of LDL Particles Ready for Prime Time? Clinical Chemistry, 52, 1643-1644. https://doi.org/10.1373/clinchem.2006.073452

[17] Muñiz, N. (1977) Measurement of Plasma Lipoproteins by Electrophoresis on Polyacrylamide Gel. Clinical Chemistry, 23, 1826-1833.

[18] Roche, D., Atger, V., Le Quang, N.T., Girard, A. and Ekindjian, O.G. (1985) Polyacrylamide Gel Electrophoresis in Quantification of High-Density Lipoprotein Cholesterol. Clinical Chemistry, 31, 1893-1895.

[19] Bañuls, C., Bellod, L., Jover, A., Martínez-Triguero, M.L., Víctor, V.M., Rocha, M. and Hernández-Mijares, A. (2012) Comparability of Two Different Polyacrylamide Gel Electrophoresis Methods for the Classification of LDL Pattern Type. Clinica Chimica Acta, 413, 251-257. https://doi.org/10.1016/j.cca.2011.09.047

[20] Mishima, Y., Ando, M., Kuyama, F., Ishioku, T. and Kihata, M. (1997) A Simple Method for Identifying Particle Size of Low-Density Lipoprotein Using PAG Electrophoresis: Comparison between LipoPhor and Lipoprint LDL Systems. Journal of Japan Atherosclerosis Society, 25, 67-70. (In Japanease) https://www.jstage.jst.go.jp/article/jat1973/25/1-2/25_1-2_67/_pdf

[21] Yoshida, A., Kodama, M., Nomura, H. and Naito, M. (2003) Classification of Lipoprotein Profile by Polyacrylamide Gel Disc Electrophoresis. Internal Medicine, 42, 244-249. https://doi.org/10.2169/internalmedicine.42.244

[22] Agouridis, A.P., Kostapanos, M.S., Tsimihodimos, V., Kostara, C., Mikhailidis, D.P., Bairaktari, E.T., Tselepis, A.D. and Elisaf, M.S. (2012) Effect of Rosuvastatin Monotherapy or in Combination with Fenofibrate or $\omega$-3 Fatty Acids on Lipoprotein Subfraction Profile in Patients with Mixed Dyslipidaemia and Metabolic Syndrome. International Journal of Clinical Practice, 66, 843-853. https://doi.org/10.1111/j.1742-1241.2012.02972.x

[23] Bando, Y., Toyama, H., Kanehara, H., Hisada, A., Okafuji, K., Toya, D. and Tanaka, N. (2016) Switching from Atorvastatin to Rosuvastatin Lowers Small, Dense LowDensity Lipoprotein Cholesterol Levels in Japanese Hypercholesterolemic Patients with Type 2 Diabetes Mellitus. Diabetes Research and Clinical Practice, 111, 66-73. https://doi.org/10.1016/j.diabres.2015.10.013

[24] Griffin, B.A. and Packard, C.J. (1994) Metabolism of VLDL and LDL Subclasses. Current Opinion in Lipidology, 5, 200-206. https://doi.org/10.1097/00041433-199405030-00007

[25] Griffin, B.A. (1997) Low-Density Lipoprotein Subclasses: Mechanisms of Formation and Modulation. Proceedings of the Nutrition Society, 56, 693-702. https://doi.org/10.1079/PNS19970069

[26] Yoshino, G., Nakano, S., Matsumoto, T., Murakami, E., Morita, T. and Kuboki, K. (2012) Rosuvastatin Reduces Plasma Small Dense LDL-Cholesterol Predominantly in Non-Diabetic Hypercholesterolemic Patients. Pharmacology \& Pharmacy, 3, 7278. https://doi.org/10.4236/pp.2012.31011

[27] Roubtsova, A., Munkonda, M.N., Awan, Z., Marcinkiewicz, J., Chamberland, A., Lazure, C., Cianflone, K., Seidah, N.G. and Prat, A. (2011) Circulating Proprotein Convertase Subtilisin/Kexin 9 (PCSK9) Regulates VLDLR Protein and Triglyceride Accumulation in Visceral Adipose Tissue. Arteriosclerosis, Thrombosis, and Vascular Biology, 31, 785-791. https://doi.org/10.1161/ATVBAHA.110.220988

[28] Tavori, H., Rashid, S. and Fazio, S. (2015) On the Function and Homeostasis of PCSK9: Reciprocal Interaction with LDLR and Additional Lipid Effects. Atherosclerosis, 238, 264-270. https://doi.org/10.1016/j.atherosclerosis.2014.12.017 
[29] Yoshino, G., Hirano, T. and Kazumi, T. (2002) Treatment of Small Dense LDL. Journal of Atherosclerosis and Thrombosis, 9, 266-275. https://doi.org/10.5551/jat.9.266

[30] Hirano, T., Ito, Y. and Yoshino, G. (2005) Measurement of Small Dense Low-Density Lipoprotein Particles. Journal of Atherosclerosis and Thrombosis, 12, 67-72. https://doi.org/10.5551/jat.12.67

[31] Ikeda, H. (1991) A Case of Symptomatic Hypobetalipoproteinemia with Unusual Distribution of Apoprotein E. Japanese Journal of Geriatrics, 28, 823-828. (In Japanese) https://doi.org/10.3143/geriatrics.28.823

[32] Mead, M.G. and Dangerfield, W.G. (1974) The Investigation of "Mid-Band" Lipoproteins Using Polyacrylamide Gel Electrophoresis. Clinica Chimica Acta, 51, 173 182. https://doi.org/10.1016/0009-8981(74)90027-8

[33] Nakada, F., Nakada, K. and Maehira, F. (1976) Purification of Human Serum Albumin and Gamma-Globulin by Using a Pellico-Electrofractionator. The PhysicoChemical Biology, 20, 119-123. (In Japanese) https://www.jstage.jst.go.jp/article/sbk1951/20/2/20_2_195/_pdf

[34] Totsuka, M., Miyashita, Y., Itoh, Y., Hashiguchi, S., Urano, Y., Watanabe, J., Tomioka, H. and Shirai, K. (1997) Properties of Fast Beta Lipoprotein on the PAG Disc Electrophoresis and Its Role in Diabetic Complications. Journal of the Japan Diabetes Society, 40, 503-511. (In Japanese) https://www.jstage.jst.go.jp/article/tonyobyo1958/40/8/40_8_503/_pdf

[35] Miyauchi, K., Kayahara, N., Ishigami, M., Kuwata, H., Mori, H., Sugiuchi, H., Irie, T., Tanaka, A., Yamashita, S. and Yamamura, T. (2007) Development of a Homogeneous Assay to Measure Remnant Lipoprotein Cholesterol. Clinical Chemistry, 53, 2138-2135. https://doi.org/10.1373/clinchem.2007.092296

[36] Yamashita, S., Ishigami, M. (2008) Efficacy of Remnant/TG Ratio, Remnant/non HDL-C Ratio Measurement Using MetaboLead Rem-C. Journal of Medicine and Pharmaceutical Sciences, 59, 429-437.

[37] Stein, E.A. and Raal, F. (2016) Future Directions to Establish Lipoprotein(a) as a Treatment for Atherosclerotic Cardiovascular Disease. Cardiovascular Drugs and Therapy, 30, 101-108. https://doi.org/10.1007/s10557-016-6654-5

[38] Blom, D.J., Hala, T., Bolognese, M., Lillestol, M.J., Toth, P.D., Burgess, L., Ceska, R., Roth, E., Koren, M.J., Ballantyne, C.M., Monsalvo, M.L., Tsirtsonis, K., Kim, J.B., Scott, R., Wasserman, S.M. and Stein, E.A. (2014) A 52-Week Placebo-Controlled Trial of Evolocumab in Hyperlipidemia. New England Journal of Medicine, 370, 1809-1819. https://doi.org/10.1056/NEJMoa1316222

[39] Raal, F.J., Stein, E.A., Dufour, R., Turner, T., Civeira, F., Burgess, L., Langslet, G., Scott, R., Olsson, A.G., Sullivan, D., Hovingh, G.K., Cariou, B., Gouni-Berthold, I., Somaratne, R., Bridges, I., Scott, R., Wasserman, S.M. and Gaudet, D. (2015) PCSK9 Inhibition with Evolocumab (AMG 145) in Heterozygous Familial Hypercholesterolaemia (RUTHERFORD-2): A Randomised, Double-Blind, Placebo-Controlled Trial. Lancet, 385, 331-340. https://doi.org/10.1016/S0140-6736(14)61399-4

[40] Evans, R.M., Hui, S., Perkins, A., Lahiri, D.K., Poirier, J. and Farlow, M.R. (2004) Cholesterol and APOE Genotype Interact to Influence Alzheimer Disease Progression. Neurology, 62, 1869-1871. https://doi.org/10.1212/01.WNL.0000125323.15458.3F

[41] Corder, E.H., Saunders, A.M., Risch, N.J., Strittmatter, W.J., Schmechel, D.E., Gaskell, P.C., Rimmler, J.B., Locke, P.A., Conneally, P.M., Schmader, K.E., Small, G.W., Roses, A.D., Haines, J.L. and Pericak-Vance, M.A. (1994) Protective Effect of Apolipoprotein E Type 2 Allele for Late Onset Alzheimer Disease. Nature Genetics, 7, 180-184. https://doi.org/10.1038/ng0694-180 


\section{Abbreviations}

$\begin{array}{ll}\text { PCSK9 } & \text { protein convertase subtilisin/kexin type } 9 \\ \text { LDL } & \text { low-density lipoprotein } \\ \text { IDL } & \text { intermediate-density lipoprotein } \\ \text { VLDL } & \text { very low-density lipoprotein } \\ \text { HDL } & \text { high-dense lipoprotein } \\ \text { sdLDL } & \text { small dense LDL } \\ \text { lbLDL } & \text { large buoyant LDL } \\ \text { L-LDL } & \text { large size LDL } \\ \text { M-LDL } & \text { medium size LDL } \\ \text { s-LDL, } & \text { small size LDL } \\ \text { vs-LDL } & \text { very small size LDL } \\ \text { PAGE } & \text { poly-acrylamide gel electrophoresis method } \\ \text { GGE } & \text { poly-acrylamide gradient gel electrophoresis method } \\ \text { CAD } & \text { coronary artery disease } \\ \text { HMG-CoA } & \text { 3-hydroxy-methylglutaryl CoA } \\ \text { Rm } & \text { relative migration } \\ \text { ApoE } & \text { apoprotein E }\end{array}$

\section{Scientific Research Publishing}

Submit or recommend next manuscript to SCIRP and we will provide best service for you:

Accepting pre-submission inquiries through Email, Facebook, LinkedIn, Twitter, etc. A wide selection of journals (inclusive of 9 subjects, more than 200 journals)

Providing 24-hour high-quality service

User-friendly online submission system

Fair and swift peer-review system

Efficient typesetting and proofreading procedure

Display of the result of downloads and visits, as well as the number of cited articles Maximum dissemination of your research work

Submit your manuscript at: http://papersubmission.scirp.org/

Or contact ojmip@scirp.org 